\title{
Technè
}

La science au service de l'histoire de l'art et de la préservation des biens culturels

48 | 2019

Les couleurs de l'Antique

\section{Mesopotamian Sculpture in Colour, edited by Astrid Nunn and Heinrich Piening, PeWe-Verlag, Gladbeck, 2020}

Annonce de parution

\section{Astrid Nunn}

\section{OpenEdition}

\section{Journals}

Édition électronique

URL : https://journals.openedition.org/techne/2783

DOI : 10.4000/techne.2783

ISSN : 2534-5168

Éditeur

C2RMF

Édition imprimée

Date de publication : 31 décembre 2019

Pagination : 133-134

ISBN : 978-2-11-152831-4

ISSN : $1254-7867$

Référence électronique

Astrid Nunn, «Mesopotamian Sculpture in Colour, edited by Astrid Nunn and Heinrich Piening. PeWe-

Verlag, Gladbeck, 2020 », Technè [En ligne], 48| 2019, mis en ligne le 01 juillet 2021, consulté le 02 avril 2022. URL : http://journals.openedition.org/techne/2783 ; DOI : https://doi.org/10.4000/techne.2783

\section{(c) (i) $\ominus$}

La revue Technè. La science au service de l'histoire de l'art et de la préservation des biens culturels est mise à disposition selon les termes de la Licence Creative Commons Attribution - Pas d'Utilisation Commerciale - Pas de Modification 4.0 International. 
Astrid Nunn

\title{
Mesopotamian Sculpture in Colour, edited by Astrid Nunn and Heinrich Piening, PeWe-Verlag, Gladbeck, 2020
}

\author{
Annonce de parution
}

\section{Le projet}

En Occident, on pense souvent que les statues antiques n'étaient pas polychromées et qu'elles avaient, dès l'origine, la couleur de la pierre. Cette idée fausse reste bien ancrée dans les mentalités malgré le nombre et la vulgarisation croissante des recherches portant sur la couleur dans l'Antiquité. Il n'y a aucune raison pour que les spectateurs de la statuaire orientale réagissent différemment. Pourtant, il ne fait maintenant plus aucun doute que les effigies humaines mésopotamiennes étaient colorées. L'absence de prise en compte de la polychromie dans ce domaine est due à la disparition quasi-totale des couleurs sur ces objets très anciens et à la difficulté de trouver des protocoles analytiques adaptés à l'étude des restes colorés et répondant aux exigences déontologiques des musées.

La monographie comblera cette lacune en proposant une restitution de la polychromie sur les statues de pierre mésopotamiennes. Outre la description de tous les aspects techniques (caractérisation des pigments et de leur mise en ouvre) et analytiques, on y trouvera plusieurs chapitres traitant de l'approche historique de l'utilisation des couleurs, de leur aspect social englobant la coloration des diverses catégories divines et humaines ainsi que du choix de la couleur pour les vêtements et la carnation. La couleur peut encoder des valeurs spécifiques qui nous amènent inévitablement à nous interroger sur la portée symbolique de celle-ci. Les réponses apportées aux questions posées se fondent non seulement sur l'enquête archéologique, mais aussi sur l'étude des textes akkadiens.

Notre objectif final est une histoire culturelle des couleurs sur les représentations humaines de la Mésopotamie.

\section{Les méthodes scientifiques}

Localiser de minuscules résidus de pigments est un véritable défi, encore plus grand que dans l'art égyptien ou classique où les couleurs peuvent être mieux préservées. Ici, les objets examinés sont restés enfouis durant des milliers d'années et ont été dès leur découverte intensément nettoyés, réparés, vernis, marqués...

Le deuxième défi consistait à trouver un protocole analytique adapté pour caractériser les pigments afin qu'il soit possible non seulement de déterminer leur nature mais aussi d'estimer leur ancienne couleur. Toutes les techniques testées sont des spectroscopies, mais seules deux d'entre elles ont été retenues : 1) La spectroscopie Raman mesure la composition moléculaire et la structure du matériau (ou de son environnement, ou encore de son degré d'oxydation), ce qui rend possible l'identification de celui-ci. La couleur n'est alors pas mesurée, mais seulement extrapolée à partir du matériau. 2) Malgré ses limites, la spectroscopie UV-Vis (ultra-violet visible) s'est finalement avérée être mieux adaptée que la spectroscopie Raman ou la spectrométrie de fluorescence de rayons $\mathrm{X}$ (XRF), d'autant que Heinrich Piening (physicien rattaché au Centre de Restauration des Châteaux de Bavière, Munich) a conçu pour cette recherche un équipement UV-Vis portable.

En conclusion, l'idéal aurait été de combiner plusieurs types de spectroscopies, idéal toutefois impossible à appliquer lors de nos missions d'étude dans les musées.

\section{Le corpus}

Au total, nous avons examiné 179 statues conservées au Vorderasiatisches Museum de Berlin, au British Museum de Londres, aux Musées Royaux d'Art et d'Histoire à Bruxelles et à l'Oriental Institute Museum de Chicago. Le corpus rend ainsi compte de l'évolution des pratiques de mise en couleur de la sculpture mésopotamienne sur une période allant d'environ 3000 à 625 avant notre ère.

\section{Les couleurs et leur emploi}

La palette des pigments est très restreinte. La catégorie du « noir » consiste en bitume et en produits carbonisés mélangés à des huiles. Tous les tons chauds, le jaune, l'orange, le 


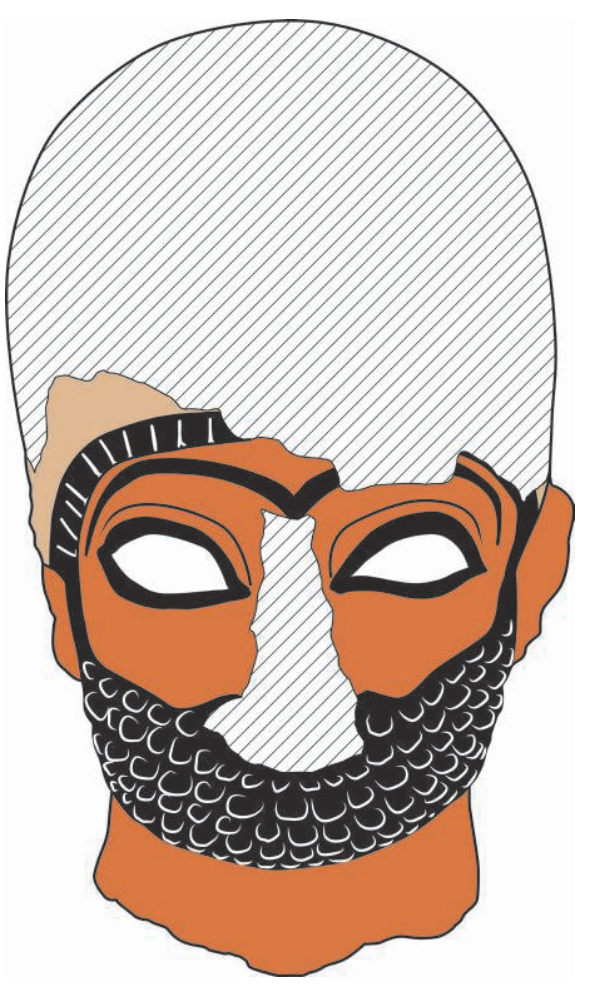

Fig. 1. Tête masculine, Ishchali (H. 4,5 cm), 1900-1750 av. J.-C., inv. A17009. Courtesy of the Oriental Institute of the University of Chicago.

jaune orangé, le rouge, le rouge brun, le marron clair et le marron foncé sont fabriqués à base d'oxydes de fer, en général de l'hématite. Les minéraux sont broyés à divers degrés ; plus le grain est fin, plus la couleur est claire. À l'exception de rares cas où l'on trouve des tons de rouge mêlés à du blanc (craie, céruse, kaolinite, gypse ?), pour dépeindre des carnations, les pigments ne sont jamais mélangés. Le blanc en tant que couleur appliquée est inhabituel. Nous n'avons décelé aucune trace ni de bleu, ni de vert.

Le noir est la couleur la mieux préservée. C'est essentiellement la couleur des cheveux et de certains détails tels les bordures des vêtements et les signes des inscriptions cunéiformes. Les tons de rouge sont moins bien conservés. À l'origine toutefois, ils dominaient l'aspect général puisque les parties principales de la statue, carnation et vêtement, étaient traitées en teintes chaudes (fig. 1). En outre, les coiffes, les bordures et les franges des vêtements, la pelisse des animaux, les ongles, les sièges (fig. 2), les inscriptions et les bases pouvaient également être « rouges».
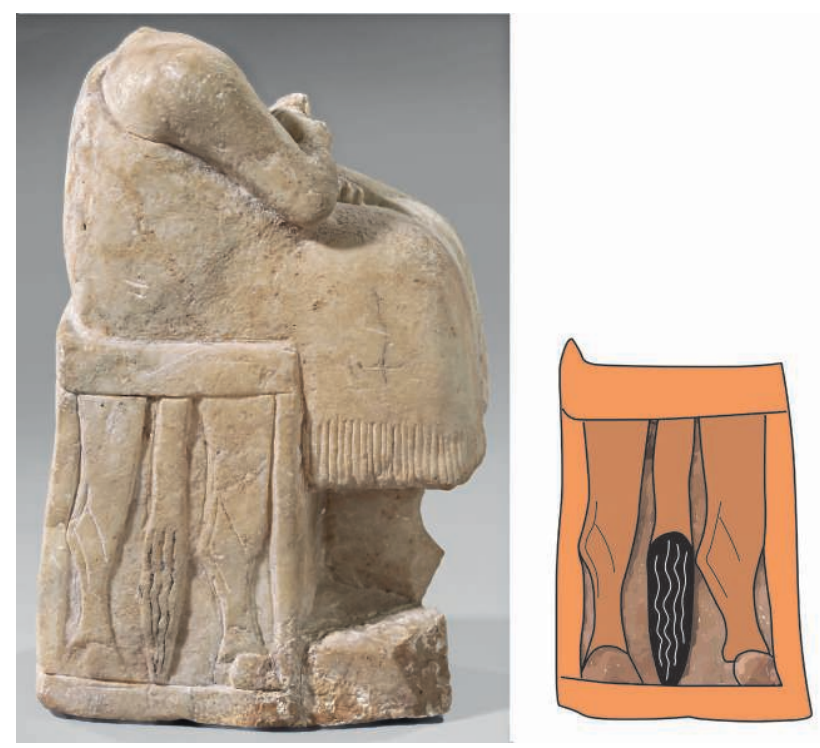

Fig. 2. Femme assise sur un tabouret, Assur (H. $29 \mathrm{~cm})$, 2575-2300 av. J.-C., inv. VA 8145. () Staatliche Museen zu Berlin, Vorderasiatisches Museum/O. M. Teßmer.

\section{Conclusion}

Une statue devait être recouverte de couleurs en Mésopotamie, comme ce fut le cas en Égypte et en Grèce. Ainsi, les deux champs principaux de couleur, les tons de noir et de rouge, allant de pair avec éclat, intensité et luminosité, participaient de la réalité tout en ayant une connotation positive. Avec les couleurs, nous découvrons maintenant le vrai visage des statues dont la fonction était d'incarner devant les dieux la personne représentée. Les couleurs de la vie, du bien-être et de la communication étaient en Mésopotamie fortes et lumineuses. 